\title{
Die Blutkatalaseaktivität des Kindes und ihre Beeinflussung durch Erkrankungen*)
}

\author{
Von \\ E. WERNER und H. HeIDER \\ Aus der Kinderklinik der Freien Universität Berlin, Kaiserin AugusteVictoria Haus (Direktor: Prof. Dr. A. Loeschlee)
}

(Der Schriftleitung zugegangen am 26. März 1963)

\begin{abstract}
Die Annahme, daß die hohe Katalaseaktivität der Erythrozyten einen Schutz des Organismus gegen den oxydativen Abbau des Hämoglobins darstellt, gibt Anlaß zu Untersuchungen über das Verhalten der Katalaseaktivität des Blutes bei Kindern, wenn der Hämoglobin- oder Erythrozytenbestand durch verschiedene Ursachen beeinflußt wird. Eine Aktivitätsverminderung der Katalase findet sich bei Säuglingen, besonders bei Frühgeborenen und reifen Neugeborenen während der ersten Lebenstage in Korrelation zu einer Labilität des Erythrozytenstoffwechsels. Jenseits des 1. Lebensjahres bis zur Pubertät ist die Katalaseaktivität konstant. Eine Geschlechtsabhängigkeit besteht nicht. Bei Anämien im Verlauf von Staphylokokkeninfekten, Nephritiden, akuten Leukosen und bei symptomatischer Polycythämie infolge angeborener Herzfehler ist die Katalaseaktivität herabgesetzt. Die Aktivitätshemmung bei konstitutioneller hämolytischer Anämie wird durch Milzexstirpation gebessert. Bei Blutungsanämien oder Erkrankungen, bei denen wir keine Beeinflussung des roten Blutbildes fanden, ist die Katalaseaktivität im Bereich der Norm. Durch diese Ergebnisse wird die Theorie, daß die Katalase ein wesentliches Schutzferment des Hämoglobins und der Erythrozyten ist, gestützt.
\end{abstract}

The high catalase activity of exythrocytes may represent protection against the oxidative degradation of haemoglobin. We have therefore studied the catalase activity in the blood of children, in which haemoglobin or erythrocytes are in some way affected. Catalase activity is decreased in unweaned children and especially during the first day of birth in premature and mature infants, showing a correlation with an unstable erythrocyte metabolism. After the first year of life until puberty, catalase activity is constant. There is no variation with sex. In the anaemias during staphylococcal infections, nephrotic disorders, acute leucoses and symptomatic polycythaemia from inherited cardiac disorder, the catalase activity is decreased. The low activity in constitutional haemolytic anaemia is improved by removal of the spleen. In haemorrhagic anaemias or illnesses where there is no effect on the red cell pattern, the catalase activity is normal. These results support the conclusion that catalase is an essential protective enzyme for haemoglobin and erythrocytes.

Die physiologische Bedeutung der Katalase wird in der Beseitigung des im Stoffwechsel entstehenden Zellgiftes Wasserstoffperoxyd gesehen. Auffallend hoch ist der Katalasegehalt der Erythrozyten $(1,2)$. Nach Experimenten von BINGord (3, 4, 5, 6), Lemberg (7), Heubner $(8,9)$ u. a. scheint die Katalase in den Erythrozyten ein Schutzferment des Hämoglobins und seines Trägers gegen peroxydatisch wirksame Stoffe zu sein. Beim physiologischen Hämoglobinabbau sind $\mathrm{H}_{2} \mathrm{O}_{2}$ und Katalase im Gleichgewicht. Die Inaktivierung . der Katalase leitet den Abbau ein (10), denn mit der Alterung der Erythrozyten sinkt der Katalasegehalt und die Atmung steigt an $(11,12)$. Je nach Ausmaß der Katalasehemmung $(4,5,10,13)$ kann es $z u$ qualitativ und quantitativ unterschiedlichen oxydativen Veränderungen am zweiwertigen Eisen oder am Porphyrinring des Hämoglobinmoleküls kommen, wodurch Hämoglobin, Verdohämochromogene und im extremen Fall das farblose Propentdyopent entstehen $(14,15,16)$. Die Oxydation des $\mathrm{Fe}^{++} \mathrm{zu} \mathrm{Fe} \mathrm{Fe}^{+++}$ist reversibel, solange die reduzierenden Fermentsysteme im Erythrozyten intakt sind (17) odẹ Redụtionsmittel

*) Herrn Prof. Dr. G. Joppich zum 60. Geburtstag. verabfolgt werden $(9,15)$. Greift die oxydative Noxe den Eiweißbestandteil des Hämoglobins oder der Zellmembran an, dann sind Bildung HerNzscher Innenkörper und Hämolyse die Folge (9, 14, 15). - Die Hinweise auf die Bedeutung der Katalase für die Erhaltung des Hämoglobins und der Erythrozyten geben den Anlaß zu Untersuchungen über die Abhängigkeit der Katalaseaktivität des Blutes vom Alter und Geschlecht des Kindes, von Blutkrankheiten und Erkrankungen, die ohne wesentliche Veränderungen des roten Blutbildes einhergehen.

\section{Methodik}

Die Bestimmung der Katalaseaktivität des Blutes erfolgt nach dem Prinzip des optischen Tests nach WARBURG. Eine Beschreibung der Methode findet sich in dieser Zeitschrift (18).

\section{Ergebnisse}

a) Alters- und Gescblechtsverteilung

Zur Beurteilung von Abweichungen der Katalaseaktivität des Blutes von der Norm ist die Kenntnis der mit der angewandten Methode ermittelten Normalwerte erforderlich. Der Normwert $\bar{x}$ 
einer Altersgruppe resultiert aus dem Durchschnittswert der $\mathrm{KI}_{\mathrm{Hb}}$ von je 10 gesunden Kindern. Innerhalb der einzelnen Gruppe wird auf eine gleichmäßige Geschlechts- und Altersverteilung geachtet (Tabelle 1).

Tab. 1

Blutkatalaseaktivität in den ersten 10 Lebenstagen bei reifgeborenen Kindern

\begin{tabular}{|c|c|c|c|c|c|}
\hline Geschlecht & $\begin{array}{c}\text { Alter in } \\
\text { Tagen }\end{array}$ & $\mathrm{Hb} \mathrm{g} \%$ & $\mathrm{KI} \mathrm{I}_{\mathrm{Hb}}$ & $\begin{array}{c}\text { Normwert } \bar{x} \\
\text { des } \mathrm{KI}_{\mathrm{Hb}}\end{array}$ & $s \bar{x}$ \\
\hline \multirow{5}{*}{ 우 } & 3 & 19,47 & 349 & \multirow{5}{*}{334} & \\
\hline & 4 & 19,39 & 325 & & \\
\hline & 5 & 17,69 & 354 & & \\
\hline & 7 & 17,78 & 303 & & \\
\hline & 9 & 19,41 & 338 & & \\
\hline \multirow{5}{*}{$\hat{o}$} & 1 & 15,86 & 342 & \multirow{5}{*}{329} & \\
\hline & 4 & 18,74 & 320 & & \\
\hline & 8 & 21,30 & 348 & & \\
\hline & 9 & 19,35 & 284 & & \\
\hline & 10 & 17,55 & 350 & & \\
\hline$q+\hat{o}$ & $1-10$ & 18,65 & & 332 & \pm 7 \\
\hline
\end{tabular}

In Tabelle 2 sind die Normwerte $\overline{\mathrm{X}}$ der Blutkatalaseaktivität verschiedener Altersgruppen bei Kindern bis zum 14. Lebensjahre wiedergegeben. Frühgeborene haben während der ersten Lebenstage eine geringere Katalaseaktivität des Blutes als gleichaltrige Reifgeborene. Bis zum Ende des 1 . Trimenon steigt die Fermentaktivität bereits deutlich an, liegt aber noch immer unter der von Kindern jenseits des 1 . Lebensjahres. Vom 2. Lebensjaht an sind Werte erreicht, die sich bis zum 14. Lebensjahr nicht mehr signifikant ändern.

Tab. 2

Blutkatalaseaktivität in Abhängigkeit vom Lebensalter

\begin{tabular}{|c|c|c|c|c|c|}
\hline & Zahl & Alter & $\mathrm{Hb} \mathrm{g} \%$ & $\begin{array}{c}\text { Normwert } \overline{\mathbf{x}} \\
\operatorname{des} \mathrm{KI}_{\mathrm{Hb}}\end{array}$ & $\mathbf{s} \overline{\mathbf{x}}$ \\
\hline Frühgeborene & 10 & $1-10 \mathrm{Tg}$. & 17,07 & 290 & \pm 13 \\
\hline Reifgeborene & 10 & $1-10 \mathrm{Tg}$. & 18,65 & 332 & \pm 7 \\
\hline Säuglinge & 10 & $4-7$ Wo. & 13,39 & 376 & \pm 18 \\
\hline Säuglinge & 10 & 8-11 Wo. & 11,44 & 373 & \pm 15 \\
\hline Kinder & 10 & $1-3 \mathrm{~J}$. & 12,35 & 452 & \pm 14 \\
\hline Kinder & 10 & $4-6 \mathrm{~J}$. & 12,65 & 447 & \pm 14 \\
\hline Kinder & 10 & $7-10 \mathrm{~J}$. & 12,90 & 447 & \pm 15 \\
\hline Kinder & 10 & $11-14 \mathrm{~J}$. & 13,11 & 457 & \pm 16 \\
\hline
\end{tabular}

Die Tabelle 3 zeigt eine Gegenüberstellung der Katalaseaktivitätswerte von Jungen und Mädchen im Alter von 1-14 Jahren. Die sehr geringen Geschlechtsunterschiede der Katalaseaktivität bei Säuglingen (Tabelle 1) und älteren Kindern (Tabelle 3 ) sind statistisch nicht gesichert.

Tab. 3

Blutkatalaseaktivität in Abhängigkeit vom Geschlecht

\begin{tabular}{cccccc}
\hline Geschlecht & Zahl & $\begin{array}{c}\text { Alter } \\
\text { (Jahre) }\end{array}$ & Hb g\% & $\begin{array}{c}\text { Normwert } \overline{\mathbf{x}} \\
\text { des KI } I_{\mathrm{Hb}}\end{array}$ & $\mathbf{s} \overline{\mathbf{x}}$ \\
\hline \multirow{2}{*}{$\delta^{\prime}$} & 20 & $1-14$ & 12,54 & 453 & \pm 10 \\
& 20 & $1-14$ & 12,95 & 448 & \pm 11 \\
\hline
\end{tabular}

\section{b) Katalaseaktivität bei Anämien und bei symptomatischer Polycytbämie}

Die Ergebnisse bei Anämien verschiedener Genese im Vergleich zu den entsprechenden Normwerten gesunder Kinder sind in Tabelle 4 zusammengestellt. Eindrucksvolle Katalaseaktivitätserniedrigungen finden sich bei Anämien, die im Verlauf einer Glomerulonephritis, eines Staphylokokkeninfektes und bei akuter Leukose untersucht wurden. Dagegen treten keine eindeutigen Veränderungen des $\mathrm{KI}_{\mathrm{Hb}}$ bei Blutungsanämien auf.

Tab. 4

Blutkatalaseaktivität bei Anämien verschiedener Genese

\begin{tabular}{lcrccc}
\hline Krankheit & $\begin{array}{c}\text { Alter } \\
\text { (Jahre) }\end{array}$ & $\mathrm{Hb}$ g\% & $\mathrm{KI}_{\mathrm{Hb}} \begin{array}{c}\text { Normwert } \overline{\mathrm{x}} \\
\text { des } \mathrm{KI}_{\mathrm{Hb}}\end{array}$ & $\mathrm{s} \overline{\mathrm{x}}$ \\
\hline Glomerulonephritis & 5 & 11,20 & 328 & & \\
& 6 & 11,23 & 386 & & \\
& 7 & 11,13 & 360 & 451 & \pm 7 \\
& 8 & 11,23 & 383 & & \\
Staphylokokken- & 11 & 8,76 & 284 & & \\
infekt & 1 & 10,33 & 318 & & \\
akute Leukose & 3 & 10,00 & 324 & 451 & \pm 7 \\
& 4 & 4,71 & 19 & & \\
Blutungsanämie & 11 & 5,67 & 207 & 451 & \pm 7 \\
& $7 \mathrm{Tage}$ & 13,56 & 342 & 332 & \pm 7 \\
& 1 & 8,00 & 487 & 451 & \pm 7 \\
\hline
\end{tabular}

In Tabelle 5 sind bei 3 Kindern mit konstitutioneller hämolytischer Anämie die Katalaseaktivitätswerte a) unmittelbar vor der Milzexstirpation und b) nach der Operation wiedergegeben. Unter c) ist eine Kontrolluntersuchung, die nach mehreren Wochen bei einem Kind ohne Operation durchgeführtt worden ist, angeführt. Der zeitliche Abstand zwischen den Untersuchungen unter a) und denen unter b) und c) beträgt etwa 5 Wochen.

Die Blutkatalaseaktivität liegt bei der konstitutionellen hämolytischen Anämie unter dem Normalwert. Ein Anstieg der Fermentaktivität nach Milzexstirpation ist angedeutet. Dagegen bleibt der $\mathrm{KI}_{\mathrm{Hb}}$ eines Patienten, bei dem noch keine Entfernung der Milz vorgenommen werden konnte, über Wochen nahezu konstant.

Tab. 5

Blutkatalaseaktivität bei konstitutioneller hämolytischer Anämie vor und nach Milzexstirpation

\begin{tabular}{|c|c|c|c|}
\hline $\begin{array}{l}\text { Konstitutionelle } \\
\text { hämolyt. Anämie }\end{array}$ & $\begin{array}{l}\text { Alter } \\
\text { (Jahre) }\end{array}$ & $\mathrm{Hb} \mathrm{g} \%$ & $\begin{array}{c}\mathrm{KI}_{\mathrm{Hb}} \underset{\mathrm{KI}_{\mathrm{Hb}}}{\text { Normwert } \overline{\mathrm{x}}} \\
\mathrm{K}\end{array}$ \\
\hline
\end{tabular}

\begin{tabular}{|c|c|c|c|c|c|}
\hline $\begin{array}{l}\text { Vor Milz- } \\
\text { exstirpation a }\end{array}$ & 2 & 8,99 & 245 & & \\
\hline $\begin{array}{l}\text { nach Milz- } \\
\text { exstirpation b }\end{array}$ & & 10,62 & 284 & & \\
\hline $\begin{array}{l}\text { Vor Milz- } \\
\text { exstirpation a }\end{array}$ & 2 & 10,48 & 385 & 451 & \pm 7 \\
\hline $\begin{array}{l}\text { nach Milz- } \\
\text { exstirpation b. }\end{array}$ & & 9,89 & 433 & & \\
\hline $\begin{array}{l}\text { keine Milz- } \\
\text { exstirpation a }\end{array}$ & 1 & $\begin{array}{l}9,20 \\
8,41\end{array}$ & $\begin{array}{l}385 \\
386\end{array}$ & & \\
\hline
\end{tabular}


Die Tabelle 6 veranschaulicht, daß Kinder mit symptomatischer Polycythämic infolge angeborener Herzfehler eine geringere Katalaseaktivität ihres Blutes besitzen als gesunde Kinder.

Tab. 6

Blutkatalaseaktivität bei symptomatischer Polycythämie infolge angeborener Herzfehler

\begin{tabular}{rrrrr}
\hline $\begin{array}{c}\text { Alter } \\
\text { (Jahre) }\end{array}$ & $\mathrm{Hb} \mathbf{g} \%$ & $\mathrm{KI}_{\mathbf{H b}}$ & $\begin{array}{c}\text { Normwert } \\
\text { des } \mathrm{KI}_{\mathrm{Hb}}\end{array}$ & $\mathrm{s} \overline{\mathbf{x}}$ \\
\hline 4 & 24,30 & 288 & & \\
4 & 19,90 & 404 &. & \\
7 & 21,22 & 369 & 451 & \pm 7 \\
8 & 24,65 & 301 & & \\
10 & 24,91 & 365 & & \\
\hline
\end{tabular}

\section{c) Katalaseaktivität bei Erkrankungen obne Veränderungen des roten Blutbildes}

In Tabelle 7 sind mehrere Untersuchungsergebnisse bei gleichartigen Erkrankungen zu einem Durchschnittswert $\overline{\mathrm{x}}$ zusammengefaßt und dem entsprechenden Normwert $\bar{x}$, der an gesunden Kindern gewonnen worden ist, gegenübergestellt. Die Katalaseaktivität der Kinder, die an Nephrose, Pertussis oder Hepatitis erkrankten, unterscheidet sich statistisch nicht von der gesunder Kinder. Dagegen ist die Blutkatalaseaktivität bei der Poliomyelitis deutlich herabgesetzt.

Tab. 7

Blutkatalaseaktivität bei Nephrose, Pertussis, Hepatitis und Poliomyelitis

\begin{tabular}{|c|c|c|c|c|c|c|c|}
\hline & Zahl & Alter & $\mathrm{Hb} \mathrm{g} \%$ & $\begin{array}{l}\overline{\mathrm{x}} \operatorname{des} \\
\mathrm{KI} \mathrm{I}_{\mathrm{Hb}}\end{array}$ & $s \bar{x}$ & $\begin{array}{c}\text { Normwert } \bar{x} \\
\operatorname{des} \mathrm{KI}_{\mathrm{Hb}}\end{array}$ & $\bar{x} s \bar{x}$ \\
\hline Nephrose & 9 & $2-10 \mathrm{~J}$. & 13,23 & 425 & \pm 19 & 451 & \pm 7 \\
\hline Pertussis & $\begin{array}{l}6 \\
5\end{array}$ & $\begin{array}{l}8-11 \mathrm{Wo} . \\
1-3 \mathrm{~J} .\end{array}$ & $\begin{array}{l}11,67 \\
13,22\end{array}$ & $\begin{array}{l}382 \\
444\end{array}$ & $\begin{array}{l} \pm 9 \\
\pm 14\end{array}$ & $\begin{array}{l}337 \\
451\end{array}$ & $\begin{array}{l} \pm 15 \\
\pm 7\end{array}$ \\
\hline Hepatitis & 10 & $5-11 \mathrm{~J}$. & 12,50 & 429 & \pm 14 & 451 & \pm 7 \\
\hline $\begin{array}{l}\text { Polio- } \\
\text { myelitis }\end{array}$ & $\begin{array}{r}10 \\
1\end{array}$ & $\begin{array}{c}2-10 \mathrm{~J} . \\
5 \mathrm{Wo} .\end{array}$ & $\begin{array}{l}13,62 \\
11,58\end{array}$ & $\begin{array}{l}371 \\
295\end{array}$ & \pm 11 & $\begin{array}{l}451 \\
376\end{array}$ & $\begin{array}{l} \pm 7 \\
\pm 18\end{array}$ \\
\hline
\end{tabular}

\section{Diskussion}

Der entwicklungs- und altersabbängige Anstieg der Bluțkatalaseaktivität, wie wir ihn in der Reihenfolge: Frühgeborene, Reifgeborene, Säuglinge und Kleinkinder beobachtet haben, geht parallel zur Stabilisierung des Hämoglobin- ùn Erythrozytenhaụshalts. Abgesehen von quantitativen Besonderheiten des Erythrozytenbestandes und der Hämoglobinkonzentration in den einzelnen Entwicklungsphasen, findet man während der ersten Lebenswochen Abweichungen der Enzymaktivitäten in den Erythrozyten und einen wesentlichen Anteil von fetalem Hämoglobị am Gesamthämoglobin. Außer der Katalase sind u. a. die Aktivitäten der Cholinesterase, Glyoxalase und Carboanhydrase im
Erythrozyten vermindert, die Aldolase und GlutamatOxalacetat-Dehydrogenase dagegen vermehrt (19). Der Prozentsatz an fetalem Hämoglobin beträgt bei Neugeborenen etwa $60-80 \%$; bis zum 5 . Monat vermindert sich der Anteil auf etwa 10\% und wird schließlich immer mehr vom Erwachsenenhämoglobin abgelöst (20).

Alle diese Faktoren weisen auf eine gewisse Labilität des Erythrozyten- und Hämoglobinstoffwechsels hin und stimmen mit den klinischen Beobachtungen überein, daß insbesondere Frühgeborene mit ihrer sehr stark verminderten Katalaseaktivität, aber auch Reifgeborene auf bestimmte exogene Noxen, wie z. B. Naphthalin, Sulfonamide und Vitamin K-Derivate mit der Bildung von Hämiglobin, Innenkörpern und Hämolyse reagieren (21-27). Auch in vitro läßt sich eine Hemmung der Katalaseaktivität durch Vitamin K-Derivate nachweisen (28). Jenseits des Säuglingsalters erreicht die Katalaseaktivität einen Wert, der sich während des gesamten Kindesalters nicht mehr signifikant ändert. In diesem Lebensabschnitt ist unter physiologischen Bedingungen der Hämoglobinbestand nahezu stabil und die Resistenz des Hämoglobins und der Erythrozyten gegen Oxydation größer. - Eine gescblecbtsbedingte $A b-$ bängigkeit der Blutkatalaseaktivität (29) können wir für das Kindesalter nicht bestätigen. Auch die Blutstaten von Jungen und Mädchen zeigen bis zur Pubertät keine Unterschiede.

Bei Erkrankungen, die von Anämien mit hämolytischen Symptomen und einer Reduktion des Hämoglobinbestandes begleitet werden, läßt sich eine Aktivitätsminderung der Blutkatalase feststellen. Das trifft für Anämien im Verlaufe eines Staphylokokkeninfektes, einer Glomerulonephritis, akuten Leukose sowie bei konstitutioneller hämolytischer Anämie zu. - Staphylokokken besitzen reichlich Katalase (30). Sie entziehen einem Blutagar, der Katalase und Wasserstoffperoxyd enthält, zum eigenen Schutz die Katalase, während das Hämoglobin des Nährbodens durch Wasserstoffperoxyd zerstört wird (5). Bei der Glomerulonephritis kann die die Erkrankung auslösende „toxische“ Komponente die Katalase entweder direkt angreifen oder durch eine Beeinflussung des Knochenmarks, wie auch bei Staphylokokkeninfekten, in ihrer Synthese hemmen (31). Brngold zeigte, daß die Niere in der Lage ist, die Katalase an sich zu reißen, besonders wenn hämolysiertes Blut mit dem Nierenparenchym in Berührung kommt (5). Die entzündlichen Vorgänge in den Nieren bei Nephritis mit Blutaustritt aus den Glomeruli und die Steigerung des. Stoffwechsels im Nierenparenchym können für einen vermehrten Entzug der Blutkatalase durch die Niere zur Deckung ihres eigenen Bedarfs verantwortlich sein.

Die verminderte Katalaseaktivität bei akuten Leukosen dürfte in erster Linie durch eine Synthesehemmung im Knochenmark verursacht sein. Jede Beeinträchtigung der Funktion des Knochenmarks kann zu einer Hemmung der Synthese der Katalase führen, da anzunehmen ist, daß die Biosynthese der Katalase, wie auch die anderer Porphyrineisenkomplexe (32) im Knochenmark 
erfolgt. Aber auch eine direkte Einwirkung auf die Katalase ist möglich, denn bei malignen Prozessen mit Zellwucherungen werden vom Tumor bestimmte toxische Substanzen und vom Organismus proteolytische Abwehrfermente gebildet, die die Katalaseaktivität in der Leber (33) und den Erythrozyten senken (34).

Bei der konstitutionellen bämolytischen Anämie müssen die gleichen Faktoren, die zur Hämolyse führen, für die Hemmung der Katalaseaktivität verantwortlich gemacht werden. Es ist nachgewiesen, daß die Milz wesentlich beim Abbau der Häminfermente beteiligt ist (35). Die beiden Kinder, bei denen die Milz exstirpiert wurde, zeigten gleichzeitig einen Rückgang der Hämolyse und einen Anstieg der Katalaseaktivität. Ohne Milzexstirpation sahen wir bei dem 3. Kind keine Änderung der entsprechenden Befunde. Ob der verminderte Katalaseschutz der Erythrozyten an der Hämolyse beteiligt ist, ist nicht sicher. Durch Anämien nach größerem Blutverlust ohne zusätzlichen Erythrozytenabbau wird die Blutkatalaseaktivität nicht beeinflußt. Die nur sehr kurz und gering eingeschränkte Fähigkeit des Knochenmarks neben der übersteigerten Zellbildung in der Regenerationsphase genügend Hämoglobin für den einzelnen Erythrozyten zu bilden, wird sich kaum auf die Katalasesynthese auswirken, denn der Umsatz der Fermenthämine verläuft bevorzugt und schneller als der des Hämoglobins $(35,36)$.

Es liegt nahe, die Katalaseverminderung bei der symptomatischen Polycytbämie infolge angeborener Herzfehler auf eine Synthesestörung im Knochenmark zurückzuführen und als Folge einer Erschöpfung der Eisendepots zu deuten. Hiergegen spricht aber, daß dieHämoglobinbildung nicht herabgesetzt ist. Bei Polycythämie infolge Höhenanpassung ist die Katalaseaktivität sogar erhöht (37). Dies könnte die Schutzfunktion der Katalase im Erythrozytenstoffwechsel unterstreichen, denn nach HeILMEYer ist im Verlauf der Höhenanpassung der Erythrozytenabbau eingeschränkt (38). Der exogene Sauerstoffmangel erreicht aber auch nicht einen so langdauernden und schweren Grad wie der endogene bei bestimmten angeborenen Herzfehlern.

Beim nephrotischen Syndrom nach Ausschwemmung der Ödeme, bei Pertussis und Hepatitis epidemica, bei denen wir keine quantitativen Veränderungen des roten Blutbildes fanden, war auch die Blutkatalaseaktivität im Bereich der Norm. Dagegen beobachteten wir aber bei der Poliomyelitis trotz eines normalen roten Blutbildes immer eine verminderte Katalaseaktivität. Auch StAfFe (37) erwähnt eine erniedrigte Fermentaktivität bei Poliomyelitis, Heller (39) errechnete einen subnormalen $\mathrm{KI}_{\mathrm{Hb}}$. Diese Befunde weisen in die Richtung einer von japanischen Forschern aufgestellten Theorie der Katalasehemmung durch Viren (40-42). Danach wird die Katalase nicht durch die Viren zerstört, sondern nur durch Einschluß in das Virusmolekül blockiert, während das nicht mehr durch die Katalase abgebaute Wasserstoffperoxyd von den Viren zur eigenen Vermehrung benutzt wird und keine Gefahr mehr für den Erythrozyten und das Hämoglobin darstellt. Durch vorsichtige Alkalispaltung des Virusmoleküls läßt sich die Katalase wiedergewinnen (41).

Unsere Ergebnisse zeigen, daß die Katalaseaktivität des Blutes charakteristischen Schwankungen bei Änderungen im Hämoglobin- und Erythrozytenhaushalt unterliegt. Eine hohe Katalaseaktivität ist mit einer Stabilisierung des Erythrozyten- und Hämoglobinbestandes vergesellschaftet; eine geringe Katalaseaktivität begleitet einen erhöhten Abbau des Hämoglobins und der Erythrozyten. Zur Entstehung der Anämien tragen viele Faktoren bei, wie primär gehemmte Bildung der Erythrożyten und des Hämoglobins, durch Knochenmarksschädigung und Eisenmangel oder direkte Zerstörung der Erythrozyten im Blut durch Toxine usw. Zu erwägen ist aber auch, ob nicht die Katalase an der Anämisierung beteiligt ist. Durch ihre beeinträchtigte. Aktivität sind der Erythrozyt und das Hämoglobin dem oxydativen Abbau leichter ausgesetzt, sei es durch Zellperoxyde oder oxydative Noxen bakterieller oder toxischer Art. Einen weiteren Hinweis, daß die Katalase einen Oxydationsschutz für das-Hämoglobin darstellt, geben Experimente an akatalatischen Erythrozyten, die von Menschen mit kongenitaler Akatalasie stammen (43). So wird in den akatalatischen Erythrozyten durch Röntgenstrahlen, die Wasserstoffperoxyd im Organismus erzeugen (44), die Hämiglobinbildung gegenüber normalen Erythrozyten um etwa das Zwanzigfache erhöht (45).

\section{Literatur}

1. Kurokawa, H., Tohoku J. Exper. Med. 14, 520 und 539 (1929/30). - 2. Perlmann, G. E. und F. Lipmann, Arch. Biochemistry 7, 159 (1945). - 3. BrNGoLd, K., Münch. med. Wschr. 32, 1373 (1928). - 4. Bingold, K., Dtsch. Arch. klin. Med. 177, 230 (1935). - 5. Bingold, K., Aerztl. Forschg., Wörishofen 1, 41 (1947). - 6. Bingold, K. und W. Sticr,.Med. Mschr. 3, 243 (1949). - 7. Lemberg, R., B. Cortis-Jones und M. Norrie, Biochem. J. 32, 171 (1938). - 8. Heubner, W., Erg. Physiol. 43, 9 (1940). - 9. Heunner, W., Klin. Wschr. 20, 137 (1941); 21, 520 (1942). - 10. BrugsCH, J., Hämoglobin, der rote Blutfarbstoff. Georg Thieme Verlag, Leipzig (1950). - 11. LöHR, G. W., H.D. Waller, O. Karges, B. Schlegel und A. A. MülLER, Klin. Wschr. 36, 1008 (1958). - 12. Schleged, B. und
P. Kappest, Klin. Wschr. 34, 805 (1956). - 13. Duesberg, R. und D. Mohring, Abbau des Häms, S. 115, aus Heilmeyer, L. und A. Hitrmair, Hdb. ges. Hämatologie II/2, 1 MünchenBerlin: Urban \& Schwarzenberg (1959). - 14. Jung, F., Klin. Wschr. 24/25, 459 (1946/47). - 15. Opitz, H. und B. DE RudDER, Pädiatrie - Ein Lehrbuch f. Studierende u. Årzte, Springer-Verlag Berlin-Göttingen-Heidelberg (1957). - 16. SEIDE, G., Biochem. Z. 308,175 (1941). - 17. KIESE, M., Klin. Wschr. 24/25, 81 (1946/47). - 18. WERNER, E. und H. HeIDER, Diese Z., im Druck. - 19. BerKKe, K., Hämoglobin und Erythrozyten in H. WIESENER: Einführung in die Entwicklungsphysiologie des Kindes. Springer Verlag, Berlin-Göttingen-Heidelberg, im Druck. - 20. BeTKE, K., Internist, 1, 236 (1960). - 21. BeTKE, K., Der menschliche 
rote Blutfarbstoff, Springerverlag Berlin-Göttingen-Heidelberg, (1954). - 22. Haupt, H., Dtsch. med. Wschr. 85, 474 (1960). 23. Heider, H., Die Blutkatalaseaktivität im Kindesalter u. ihre Beeinflussung durch bestimmte Erkrankungen u. Vitamin KPräparate, Med. Diss., Berlin (1962). - 24. KüNzER, W., E. Ambs und D. SCHNEIDER, Klin. Wschr. 31,617 (1953). - 25. NirsCh, K., Klin. Wschr. 35, 363 (1957). - 26. WrLLr, H., Helvet. paediatr. acta 11, 325 (1956). - 27. WILLI, H., M. VeST und O. KäSER, Gynaecologia 147, 481 (1959). - 28. WERNER, E. und H. HEIDER, Mschr. Kinderhk. 108, 102 (1960). - 29. Schürz, E., Uber die Katalaseaktivität d. Säuglingsblutes u. ihre Bestimmung mittels Polarographie, Diss., Würzburg (1954). - 30. Fujrtı, A. und T. Kodama, Biochem. Z. 232, 20 (1931). - 31. Bücharann, P. und R. Stodtaeister, Dtsch. Arch. klin. Med. 190, 487 (1943). 32. Salomon, K., J. E. Richmond und K. J. Altmann, J. Biol. Chemistry 196, 463 (1952). - 33. Greenfield, R. E., J. Nat. Cancer Inst., Wash. 11, 997 (1951). - 34. Yamagata, S. und
T.NAkıo, Tohoku J.Exper.Med. 57, 93 (1952/53). - 35. Sticri, W., Gallenfarbstoffe, Stoffivechsel, S. 237, aus BüchNer, F., E. LETrERER und F. Roulet: Hdb. allg. Pathologie IV, 2/Stoffivechsel II, Springer-Verlag, Berlin-Göttingen-Heidelberg (1957). - 36. Schultze, M. O. und K. A. Kuiken, J. Biol. Chemistry 137, 727 (1941). - 37. Staffe, A., Ann. paediatr., Basel 175, 77 (1950). 38. Heilmeyer, L., K. Recknagel und L. Albus, Zschr. exper. Med. 90. 573 (1933). - 39. Heller, H., Röntgen-Laborat. praxis, Stuttgart, $X, 183$ (1957). - 40. Yamifujr, K. und F. Yoshihara, Biochem. Z. 316, 74 (1944). - 41. Yamafuji, K., K. So und K. Nagano, Biochem. Z. 315, 405 und 411 (1943). - 42. YamaFujr, K., K. So und K. Soo, Biochem. Z. 311, 203 (1941). 43. Aebi, H., Dtsch. med. J. 11, 314 (1926). - 44. WarburG, O., K. GAWEHN und A.W. GerssLER, Naturwissenschaften 8, 192 (1958). - 45. Aebi, H., J. P. Heiniger und H. Suter, Experientia, Basel 18, 129 (1962).

Priv. Doz. Dr. E. Werner

Universitäts-Kinderklinik der Freien Universität Berlin Kaiserin Auguste Victoria Haus

1 Berlin 19, Heubnerweg 6

\title{
Corticoid-Abbau durch faecale Wirkstoffe im Urin letal bestrahlter Meerschweinchen
}

\author{
Von \\ C. WINKLER und H. SCHORN
}

Aus der Nuclearmedizinischen Abteilung in der Cbirurgischen Universitäts-Klinik Bonn (Direktor: Prof. Dr. A. Gütgemann)

(Der Schriftleitung zugegangen am 20. April 1963)

\begin{abstract}
Im Rahmen strahlenbiologischer Untersuchungen ist die Corticoid-Ausscheidung von wesentlichem Interesse. $\mathrm{Da}$ beim akuten Strahlensyndrom Diarrhoen auftreten, kann der ausgeschiedene Urin mit Faecalien in Berührung kommen. Diese verursachen eine Umwandlung des Cortisols, die zu falschen Rückschlüssen auf den Cortisolstoffwechsel führen können. Über Art und Grad derartiger Veränderungen wird an Hand experimenteller Untersuchungen berichtet.

Corticoid excretion is of great interest in relation to biological radiation studies. Since diarthoea occurs in acute radiation syndrome, the excreted urine can be contaminated with faeces. This causes a conversion of the cortisol and can give rise to false impressions of cortisol metabolism. The type and degree of these changes are reported on the basis of experimental results.
\end{abstract}

Die Corticosteroide sind für den Verlauf der akuten Strahlenkrankheit von wesentlicher Bedeutung $(1,2)$. In zahlreichen Arbeiten ist daher über SteroidhormonUntersuchungen bei strahlenbiologischen Experimenten berichtet worden. Die zum Problem der Nebennierenfunktion nach Strahlenbelastung mitgeteilten Befunde erscheinen jedoch uneinheitlich und zum Teil widersprüchlich. Dies ist $\mathrm{u}$. E. auf die vielfach nicht vergleichbaren Versuchsanordnungen sowie in manchen Fällen auf unzweckmäßige Untersuchungstechnik und Interpretation der Ergebnisse zurückzuführen. Im Rahmen eigener experimenteller Untersuchungen haben wir uns bemüht, Produktionsrate und Stoffwechsel des Cortisols nach Strahlenbelastungen des Organismus systematisçh zu erfassen und dabei Fehlerquellen soweit wie möglich auszuschalten. Zur Klärung spezieller Probleme, über die andernorts berichtet wird $(3,4)$, wurde Meerschweinchen $\mathrm{C}^{14}$ markiertes Cortisol i. v. injiziert und die Ausscheidung des Hormons und seiner Metaboliten im Urin bestimmt. $\mathrm{Da}$ es bei den Tieren im fortgeschrittenen Stadium der Strahlenkrankheit zu Diarrhoen kommt, war der aufgefangene Urin häufig durch Faecalien verunreinigt. Diese Tatsache gab Veranlassung, zu untersuchen, ob bzw. in welcher Weise die Faeces eine Veränderung der Corticoide (nach der Excretion in vitro) bewirken. Dies erschien von wesentlichem Interesse zur Vermeidung falscher Rückschlüsse auf den Hormonstoffwechsel in vivo.

Methodik: Zur Untersuchung evt. Einwirkungen der im Urin befindlichen Faeces auf das ausgeschiedene Cortisol wählten wir folgende Versuchanordnung:

$10 \mathrm{~m} l$ Urin wurden mit $0,1 \mu \mathrm{c} C^{14}$-Cortisol in $1 \mathrm{~m} l$ physiologischer $\mathrm{NaCl}=$ Lösung versetzt. Dieser Ansatz wurde in zwei Protionen. unterteilt. Zur ersten Portion wurde etwa $1 \mathrm{~g}$ Faeces eines diarrhoischen Tieres gegeben. Diese sowie die unbehandelte zweite Portion kamen nach 24-stdg. Aufbewahrung bei Zimmettemperatur in gleicher Weise zur Aufarbeitung. - Nach ztvcimaligem Ausschütteln mit der dreifachen Menge Äthylacetat wurde die 